\title{
Deterioration of pre-existing hemiparesis due to injury of the ipsilateral anterior corticospinal tract
}

\author{
Sung Ho Jang and Hyeok Gyu Kwon
}

\begin{abstract}
Background: The anterior corticospinal tract (CST) has been suggested as one of the ipsilateral motor pathways, which contribute to motor recovery following stroke. In this study, we report on a patient who showed deterioration of pre-existing hemiparesis due to an injury of the ipsilateral anterior CST following a pontine infarct, as evaluated by diffusion tensor tractography (DTT).

Case presentation: A 55-year-old male patient showed quadriparesis after the onset of an infarct in the right pontine basis. He had history of an infarct in the left middle cerebral artery territory 7 years ago. Consequently, he showed right hemiparesis before onset of the right pontine infarct. Following this, his right hemiparesis deteriorated whereas his left hemiparesis newly developed. The DTTs for whole CST of the right hemisphere in the patient and both hemispheres in control subjects descended through the known CST pathway. By contrast, the DTT for the left whole CST of the patient showed a complete injury finding. The DTTs for the anterior CST of control subjects passed through the known pathway of the CST from cerebral cortex to medulla and terminated in the anterior funiculus of the upper cervical cord. However, the DTT for right anterior CST in the patient showed discontinuation below the right pontine infarct.
\end{abstract}

Conclusion: It appeared that the deterioration of the pre-existing right hemiparesis was ascribed to an injury of the right anterior CST due to the right pontine infarct.

Keywords: Lateral corticospinal tract, Anterior corticospinal tract, Diffusion tensor imaging, Pontine infarct, Hemiparesis

\section{Background}

The corticospinal tract (CST) is the major neuronal pathway that mediates voluntary movements in the human brain [1,2]. The CST is generally divided into the crossed lateral CST and the uncrossed anterior CST. The anterior CST primarily innervates the musculature of the trunk and proximal extremities [1,2]. The anterior CST is considered to be one of the ipsilateral motor pathways from the unaffected motor cortex to the affected extremities, which contribute to motor recovery following stroke incidents [3].

Diffusion tensor tractography (DTT), which is derived from diffusion tensor imaging (DTI), allows for the visualization and investigation of neural tracts in three dimensions [4]. Since the introduction of DTI, DTT for

\footnotetext{
* Correspondence: khg0715@hanmail.net

Department of Physical Medicine and Rehabilitation, College of Medicine, Yeungnam University, 317-1, Daemyung dong, Namku, Taegu 705-717, Republic of Korea
}

(c) 2013 Jang and Kwon; licensee BioMed Central Ltd. This is an Open Access article distributed under the terms of the Creative Commons Attribution License (http://creativecommons.org/licenses/by/2.0), which permits unrestricted use, distribution, and reproduction in any medium, provided the original work is properly cited. the whole CST has been popular in the neuroscience field, but the identification of the anterior CST from whole CST has become possible recently [5-11].

In this study, we report on a patient who showed deterioration of pre-existing hemiparesis due to an injury of the ipsilateral anterior CST following a pontine infarct, as evaluated by DTT.

\section{Case presentation}

Ethics statement

All subjects provided signed, informed consent and our institutional review board approved the study protocol.

One patient and six right-handed sex-matched control subjects (6 male; mean age: 55.7 years, range: 51-58) with no history of neurologic disease participated in this study. A 55-year-old, right-handed male was consulted to the rehabilitation department of our university hospital for his quadriparesis. Motor function was evaluated using the Medical Research Council score (MRC) [12]: 
0 , no contraction; 1 , palpable contraction but no visible movement; 2 , movement without gravity; 3 , movement against gravity; 4, movement against a resistance lower than the resistance overcome by the healthy side; 5 , movement against a resistance equal to the maximum resistance overcome by the healthy side. The patient was diagnosed as having an infarct in the right pontine basis 3 weeks earlier at the neurology department of the same university hospital. He had history of an infarct in the left middle cerebral artery (MCA) territory 7 years ago. At that time, the motor function of his right extremities had recovered to the extent that he was able to walk with limping gait pattern independently and was able to move his right arm partially. After the onset of the right pontine infarct, his right extremities showed complete weakness (MRC; 0) although his left extremities showed partial weakness (MRC; 2-4). Brain MRI taken 3 weeks after the right pontine infarct showed an old infarct in the left MCA territory, involving the whole CST area at the corona radiata and a new infarct in the right pontine basis involving the CST area (Figure 1-A).

\section{Diffusion tensor tractography}

A 6-channel head coil on a 1.5 T Philips Gyroscan Intera (Philips, Best, Netherlands) with single-shot echo-planar imaging was used for acquisition of DTI data. For each

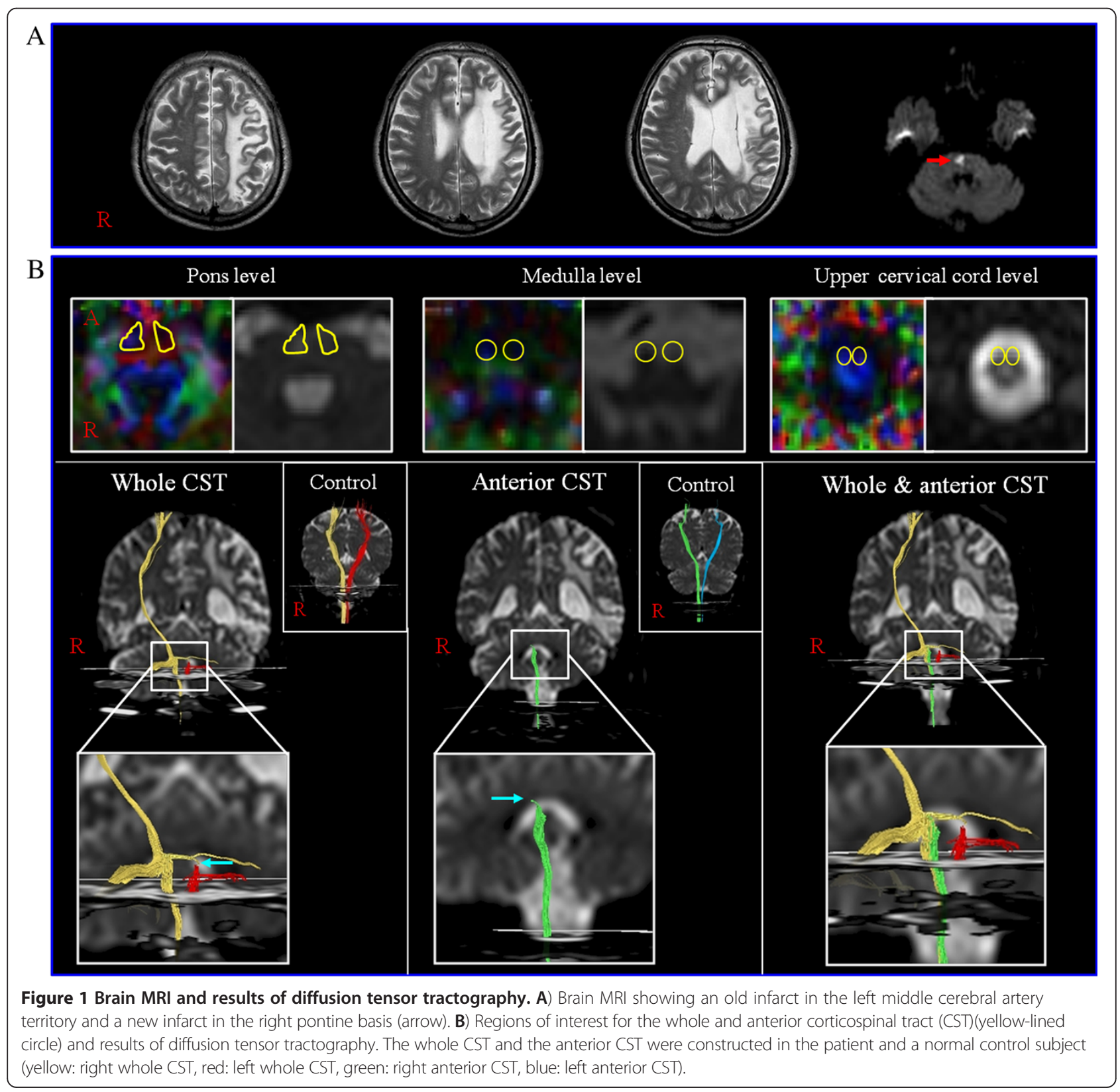


of the 32 non-collinear diffusion sensitizing gradients, we acquired 70 contiguous slices parallel to the anterior commissure-posterior commissure line. We scanned from the cortex to the middle of the second cervical vertebra body. Imaging parameters were as follows: acquisition matrix $=96 \times 96$; reconstructed to matrix $=192 \times$ 192 matrix; field of view $=240 \times 240 \mathrm{~mm}^{2}$; $\mathrm{TR}=$ $10,398 \mathrm{~ms}$; TE = $72 \mathrm{~ms}$; parallel imaging reduction factor (SENSE factor) $=2$; EPI factor $=59 ; b=1000 \mathrm{~s} / \mathrm{mm}^{2}$; $\mathrm{NEX}=1$; and a slice thickness of $2.5 \mathrm{~mm}$ (acquired isotropic voxel size $2.5 \times 2.5 \times 2.5 \mathrm{~mm}^{3}$ ). Fiber tracking was performed using the FACT algorithm implemented within the DTI task card software $[4,13]$. The whole CSTs were determined by selection of fibers passing through two regions of interest (ROIs), which were placed on the CST area of the pons and upper medulla on the color maps $[14,15]$. By contrast, for reconstruction of the anterior CST, three ROIs were placed on the CST area of the pons and upper medulla (anterior blue color) and an additional ROI was placed on the anterior funiculus of the upper cervical cord on the color maps [16]. Termination criteria was fractional anisotropy (FA) $<0.2$ and an angle change $>30^{\circ}$, as determined by a previous study on the optimal tractability threshold of FA $[10,17]$.

The DTTs for whole CSTs of the right hemisphere in the patient and both hemispheres in the control subjects originated from the primary sensori-motor cortex and descended through the medullary pyramid along the known CST pathway. By contrast, the DTT for left whole CST of the patient showed a Wallerian degeneration to the left pons with discontinuation. The DTTs for anterior CSTs of both hemispheres in control subjects originated from the primary sensorimotor cortex and passed through the known pathway of the CST from the cerebral cortex to the medulla. They then terminated in the anterior funiculus of the upper cervical cord. However, the DTT for the right anterior CST of the patient showed discontinuation below the right pontine infarct (Figure 1-B).

\section{Discussion}

In the current study, we evaluated the whole and anterior CSTs in a quadriparetic patient with a new right pontine infarct and an old left MCA infarct. According to our results, it appeared that the deterioration of the preexisting right hemiparesis was ascribed to the injury of the right anterior CST following the new right pontine infarct, for the following reasons. First, the patient had been diagnosed with an infarct in the left MCA territory 7 years ago, involving whole CST in the left corona radiata [18]. Before the onset of the new right pontine infarct, the patient was able to walk independently and move his right arm partially. However, after the onset of the new infarct in the right pontine basis, the motor function of his right extremities deteriorated to complete weakness (MRC: 0), even though his left extremities showed partial weakness (MRC: $2-4)$. We presumed that some neural tract other than the left whole CST had been responsible for the partial motor function of the right extremities before the onset of new right pontine infarct. Considering that the new infarct was located in the CST area of the right pontine basis, the right anterior CST appeared to be the most plausible motor tract for deterioration of pre-existing right hemiparesis $[14,19]$. We confirmed the injury of the right anterior CST to be due to the pontine infarct by DTT.

Little is known about the ipsilateral motor pathway that causes deterioration of pre-existing hemiparesis in stroke patients [20]. In 2003, Ago et al. reported that a 59 year-old patient experienced deterioration of the already existing left hemiparesis that had resulted from a previous right putaminal hemorrhage due to a lacunar infarct in the left corona radiata [20]. They suggested that the new infarct in the left corona radiata caused damaged the uncrossed ipsilateral motor pathway, which had been responsible for the motor function of the left extremities, causing further deterioration of the pre-existing hemiparesis. However, they could not clarify the identity of the ipsilateral motor pathway of that patient.

\section{Conclusion}

In conclusion, we showed a patient who showed deterioration of pre-existing hemiparesis by an injury of the ipsilateral anterior CST following a pontine infarct. As far as we know, this is the first DTT study to demonstrate ipsilateral motor weakness by an injury in the anterior CST of stroke patients. Several limitations of this study should be considered. First, DTI could lead to both false positive and negative throughout the white matter of brain because of complex fiber configurations [21]. Second, because it is a case report, the results of this study are limited to generalizations. Further studies to overcome these limitations should be encouraged.

\section{Consent}

Written informed consent was obtained from the patient for publication of this case report and accompanying images. A copy of the written consent is available for review by the Editor-in-Chief of this journal.

\section{Abbreviations}

CST: Corticospinal tract; DTI: Diffusion tensor imaging; DTT: Diffusion tensor tractography; FA: Fractional anisotropy; MCA: Middle cerebral artery; MRC: Medical research council; ROI: Region of interest.

\section{Competing interests}

The authors declare that they have no competing interests. 


\section{Authors' contributions}

SHJ: conceiving and designing the study, funding, data acquisition, manuscript development and manuscript writing. HGK: manuscript development, data acquisition, manuscript writing and manuscript authorization. Both authors read and approved the final manuscript.

\section{Acknowledgements}

"This research was supported by Basic Science Research Program through the National Research Foundation of Korea(NRF) funded by the Ministry of Education, Science and Technology(2012R1A1A4A01001873)".

Received: 13 May 2012 Accepted: 20 May 2013

Published: 29 May 2013

\section{References}

1. Davidoff RA: The pyramidal tract. Neurology 1990, 40(2):332-339.

2. York $\mathrm{DH}$ : Review of descending motor pathways involved with transcranial stimulation. Neurosurgery 1987, 20(1):70-73.

3. Jang $\mathrm{SH}$ : A review of the ipsilateral motor pathway as a recovery mechanism in patients with stroke. NeuroRehabilitation 2009, 24(4):315-320.

4. Mori S, Crain BJ, Chacko VP, van Zijl PC: Three-dimensional tracking of axonal projections in the brain by magnetic resonance imaging. Ann Neurol 1999, 45(2):265-269.

5. Kwon HG, Lee DG, Son SM, Byun WM, Hong CP, Lee DH, Kim S, Jang SH: Identification of the anterior corticospinal tract in the human brain using diffusion tensor imaging. Neurosci Lett 2011, 505(3):238-241.

6. Guye M, Parker GJ, Symms M, Boulby P, Wheeler-Kingshott CA, Salek-Haddadi A, Barker GJ, Duncan JS: Combined functional MRI and tractography to demonstrate the connectivity of the human primary motor cortex in vivo. Neuroimage 2003, 19(4):1349-1360.

7. Lee SK, Kim DI, Kim J, Kim DJ, Kim HD, Kim DS, Mori S: Diffusion-tensor MR imaging and fiber tractography: a new method of describing aberrant fiber connections in developmental CNS anomalies. Radiographics 2005, 25(1):53-65. discussion 66-58.

8. Cho SH, Kim SH, Choi BY, Kang JH, Lee CH, Byun WM, Jang SH: Motor outcome according to diffusion tensor tractography findings in the early stage of intracerebral hemorrhage. Neurosci Lett 2007, 421(2):142-146.

9. Wakana S, Jiang H, Nagae-Poetscher LM, van Zijl PC, Mori S: Fiber tract-based atlas of human white matter anatomy. Radiology 2004, 230(1):77-87.

10. Kunimatsu A, Aoki S, Masutani Y, Abe O, Hayashi N, Mori H, Masumoto T, Ohtomo K: The optimal trackability threshold of fractional anisotropy for diffusion tensor tractography of the corticospinal tract. Magn Reson Med Sci 2004, 3(1):11-17.

11. Kwon HG, Lee DG, Son SM, Byun WM, Hong CP, Lee DH, Kim S, Jang SH: Identification of the anterior corticospinal tract in the human brain using diffusion tensor imaging. Neurosci Lett 2011, 505(3):238-241.

12. Council MR: Aids to the examination of the peripheral nervous system. London: HMSO; 1976.

13. Stieltjes B, Kaufmann WE, van Zijl PC, Fredericksen K, Pearlson GD, Solaiyappan M, Mori S: Diffusion tensor imaging and axonal tracking in the human brainstem. Neuroimage 2001, 14(3):723-735.

14. Hong JH, Son SM, Jang SH: Somatotopic location of corticospinal tract at pons in human brain: a diffusion tensor tractography study. Neuroimage 2010, 51(3):952-955.

15. Kwon HG, Hong JH, Lee MY, Kwon YH, Jang SH: Somatotopic arrangement of the corticospinal tract at the medullary pyramid in the human brain. Eur Neurol 2011, 65(1):46-49.

16. Naidich TP, Duvernoy HM: Duvernoy's atlas of the human brain stem and cerebellum: high-field MRl: surface anatomy, internal structure, vascularization and 3D sectional anatomy. Wien; New York: Springer; 2009.

17. Kwon $\mathrm{HG}$, Jang $\mathrm{SH}$ : Compression of bilateral corticospinal tracts by bilateral pontine hemorrhage: diffusion tensor tractography study. Neural Regen Res 2011, 6(4):317-320

18. Jang SH: A review of corticospinal tract location at corona radiata and posterior limb of the internal capsule in human brain. NeuroRehabilitation 2009, 24(3):279-283.

19. Jang SH: Somatotopic arrangement and location of the corticospinal tract in the brainstem of the human brain. Yonsei Med J 2011, 52(4):553-557.
20. Ago T, Kitazono T, Ooboshi H, Takada J, Yoshiura T, Mihara F, Ibayashi S, lida M: Deterioration of pre-existing hemiparesis brought about by subsequent ipsilateral lacunar infarction. J Neurol Neurosurg Psychiatry 2003, 74(8):1152-1153.

21. Yamada K: Diffusion tensor tractography should be used with caution. Proc Natl Acad Sci U S A 2009, 106(7):14. author reply E15.

doi:10.1186/1471-2377-13-53

Cite this article as: Jang and Kwon: Deterioration of pre-existing hemiparesis due to injury of the ipsilateral anterior corticospinal tract. BMC Neurology 2013 13:53.

\section{Submit your next manuscript to BioMed Central and take full advantage of:}

- Convenient online submission

- Thorough peer review

- No space constraints or color figure charges

- Immediate publication on acceptance

- Inclusion in PubMed, CAS, Scopus and Google Scholar

- Research which is freely available for redistribution 